\title{
Cooption and Specialization of Endothelin Signaling Pathways Drove Elaboration of the Neural Crest in Early Vertebrates
}

Tyler A. Square ${ }^{1,2,}$, David Jandzik ${ }^{1,3,4}$, James L. Massey ${ }^{1}$, Marek Romášek ${ }^{1}$, Haley P. Stein ${ }^{1}$, Andrew W. Hansen ${ }^{1}$, Amrita Purkayastha ${ }^{1}$, Maria V. Cattell ${ }^{1}$, Daniel M. Medeiros ${ }^{1 *}$

1 Department of Ecology and Evolutionary Biology, University of Colorado, Boulder, CO 80309, USA

2 Current address: Department of Molecular and Cellular Biology, University of California, Berkeley, CA 94720, USA

3 Department of Zoology, Charles University in Prague, Prague, 128 44, Czech Republic

4 Department of Zoology, Comenius University in Bratislava, Bratislava, 84215, Slovakia

* Author for correspondence 


\begin{abstract}
The neural crest $(\mathrm{NC})$ is a vertebrate-specific embryonic tissue that forms an array of clade-defining adult features. A key step in the formation of these diverse derivatives is the partitioning of NC cells into subpopulations with distinct migration routes and potencies ${ }^{1}$. The evolution of these developmental modules is poorly understood. Endothelin (Edn) signaling is unique to vertebrates, and performs various functions in different NC subpopulations $s^{2-5}$. To better understand the evolution of NC patterning, we used CRISPR/Cas9-driven mutagenesis to disrupt Edn receptors, ligands, and DIx transcription factors in the sea lamprey, Petromyzon marinus. Lampreys and modern gnathostomes last shared a common ancestor 500 million years $a g 0^{6}$. Thus, comparisons between the two groups can identify deeply conserved and divergent features of vertebrate development. Using Xenopus laevis to facilitate side-by-side analyses, we show here that lamprey and gnathostomes display fundamental differences in Edn signaling function. Unlike gnathostomes, both lamprey Ednrs cooperate during oropharyngeal skeleton development. Furthermore, neither paralog regulates hand transcription factors, which are required for mandible development in gnathostomes. We also identify conserved roles for Edn signaling in $d l x$ gene regulation, pigment cell, and heart development. Together our results illustrate the stepwise neofunctionalization and specialization of this vertebrate-specific signaling pathway, and suggest key intermediate stages in the early evolution of the NC.
\end{abstract}




\section{Introduction}

The proper migration, patterning, and differentiation of most NC subpopulations requires Edn signaling. In model gnathostomes, Edn ligands secreted by oropharyngeal epithelia are bound by Edn receptors (Ednrs) expressed by NC cells. In zebrafish and mouse, disruption of edn1 or edn receptor A (ednra) results in a hypomorphic pharyngeal skeleton, skeletal element fusions, and ventral-to-dorsal transformations of oropharyngeal cartilages and bones ${ }^{2,3,7-10}$. In zebrafish, the increased dorsoventral symmetry and lack of a jaw joint causes a 'sucker' phenotype reminiscent of modern agnathans ${ }^{2}$. In both mouse and zebrafish, the skeletal phenotype of edn1/ednra mutants appears due to reduced expression of $d l x$ and hand genes in cranial NC cells ${ }^{3,11}$. In non-skeletogenic NC, loss of edn3 or ednrb causes aberrant migration and/or loss of pigment cells $s^{5,12,13}$. In mammals, these defects are accompanied by disruptions in NC-derived enteric neuron development ${ }^{14}$.

Lamprey expresses edn, ednr, $d l x$, and hand in patterns similar to their gnathostome cognates ${ }^{15-17}$ though lamprey and gnathostome NC derivatives differ substantially. In addition to lacking jaws, the lamprey oral skeleton consists of a specialized pumping organ made of a chondroid tissue called mucocartilage ${ }^{18-20}$ (Fig. 1A, Supplemental Fig. 1A and B). In the posterior pharynx, the branchial skeleton is a network of cell-rich hyaline cartilage bars, and a ventral mass of mucocartilage ${ }^{18-20}$. In the trunk, the lamprey PNS lacks sympathetic ganglia, Schwann cells, and vagal NC-derived enteric neurons ${ }^{18}$. These differences, and the unclear phylogenetic relationships between gnathostome and lamprey edn and $d l x$ homologs, have led to speculation that these genes acquired new roles in patterning the $\mathrm{NC}$ of stem gnathostomes $^{17,21}$.

\section{Results and Discussion}

To test this idea, we optimized a method for efficient Cas9-mediated mutagenesis in the sea lamprey ${ }^{22}$ and used it to disrupt the function of ednrs, edns, and dlxs. Recent assembly of the sea lamprey germline genome ${ }^{23}$ supports previous reports that lamprey has one ednra and one ednrb, and six edns, ednA- $F^{17,24}$. Because multiple, possibly redundant edns are coexpressed in lamprey embryos, we first mutagenized lamprey ednrs. Targeting two unique protein-coding sequences to control for off-target effects (Supplemental Tab. 1), we found that 
Cas9-mediated F0 mutation of ednra ( $\Delta$ ednra) resulted in a hypomorphic pharyngeal skeleton with gaps in the branchial basket, ectopic melanophores, and heart edema (Fig. 1B,C,E,F, Supplemental Figs. 1C, 2, and 3). While the $\Delta$ ednra phenotype resembles gnathostome ednra/edn 1 mutants, including the ectopic pigment cells ${ }^{25}$, it differs from the reported effects of an Edn signaling inhibitor ${ }^{26}$, likely reflecting the specificity of CRISPR/Cas9.

In gnathostomes, Ednra/Edn1 signaling acts, in part, by regulating the expression of $d l x$ paralogs in the intermediate pharynx and hand genes in the ventral pharyn $x^{3,11,27}$. We asked if lamprey ednra regulates these genes in lamprey NC cells. Despite divergent histories of $d l x$ duplication and loss ${ }^{28}$, lamprey $\Delta$ ednra larvae exhibited reduced $d l x$ expression in the intermediate domain (Fig. 1H,I,J,L,M, Supplemental Fig. 4) though ventral hand expression persisted (Fig. 10 and P). To ensure that this difference was not due to the mosaicism of Cas9mediated F0 mutagenesis, we created $\Delta e d n r a$ and $\Delta e d n 1$ Xenopus laevis larvae. As in other gnathostomes, we observed a hypomorphic pharyngeal skeleton and a loss of the jaw joint that correlated to a loss of intermediate $d l x$, and ventral hand, expression (Supplemental Fig. 5). These data suggest Edn-dependent intermediate domain expression of $d l x$ was likely present in the lamprey/gnathostome common ancestor, while Edn-dependent expression of hand is unique to gnathostomes.

Sea lamprey ednrs are broadly coexpressed in skeletogenic NC, a pattern not observed in any gnathostome, though some gnathostomes briefly express ednrb1 in these cells ${ }^{24}$. This raised the possibility that ednrb and ednra both function in lamprey pharyngeal skeleton development. We thus used three separate sgRNAs to mutagenize ednrb alone ( $\Delta e d n r b)$, and together with ednra $(\Delta e d n r a+b)$. We found that, like gnathostome ednrb/edn3 mutants, lamprey $\Delta e d n r b$ and $\Delta e d n r a+b$ individuals have severe reductions in melanophores, the only pigment cells discernible in lab-raised larvae (Supplemental Fig. 6). However, unlike any reported gnathostome ednrb mutants, alcian blue staining revealed mild skeletal defects in $\Delta$ ednrb larvae (Supplemental Figs. 1D, 6B). Furthermore, $\Delta e d n r a+b$ larvae had losses and reductions of skeletal elements that were more severe than either single mutant (Fig. 1D and G, Supplemental Figs. 1E and 6C). Intermediate $d l x$ expression was also more reduced in $\Delta e d n r a+b$ larvae than in $\Delta e d n r a$ or $\Delta e d n r b$ larvae, though $\Delta e d n r b$ individuals showed no apparent $d l x$ reduction (Fig. $1 \mathrm{~K}$ and N, Supplemental Fig. 4). In contrast, expression of hand in 
the ventral domain remained in $\Delta e d n r b$ and $\Delta e d n r a+b$ individuals (Fig. 1 Q, Supplemental Fig. 6). These results demonstrate cooperative roles for lamprey ednra and ednrb in lamprey skeletogenic NC, and confirm divergence of ventral pharyngeal NC specification between gnathostomes and lamprey.

To better understand the function of Edn signaling in lamprey NC, we analyzed the expression of several cranial NC markers in $\Delta e d n r a, \Delta e d n r b$, and $\Delta e d n r a+b$ embryos and larvae. $t w i s t A^{29}$ and soxE2 $2^{30}$ expression at st. T22-23 in $\Delta$ ednra $+b$ embryos suggests that the specification and initial migration of cranial NC cells is mostly normal in $\Delta$ ednr individuals (Fig. $2 \mathrm{~A}$ and $\mathrm{B})$. In T26.5 $\Delta$ ednra and $\Delta$ ednrb larvae, $m y c^{29}, I D^{31}$, soxE $1^{32}$, $t$ wist $A^{29}$, and $m s \times B^{15}$ expression NC also persisted in most postmigratory NC, further indicating largely normal cranial NC development, though subtle migration defects are also possible (Fig. 2C and D, Supplemental Fig. 7). In contrast, at T26.5, both $\Delta$ ednra and $\Delta$ ednrb larvae displayed clear reductions in soxE $2^{30}$ expression in the forming branchial bars and lecticanA, an aggrecan homolog, in the branchial bars and differentiating mucocartilage (Fig. 2E-G,I-K, Supplemental Fig. 1G-K), though with different penetrance (Supplemental Tab. 2). Similar reductions in soxE2 and lecticanA were observed in $\Delta e d n r a+b$ larvae, which also displayed reductions in $t w i s t A$ and soxE1, and localized loss of ID transcripts in oral mucocartilage precursors (Supplemental Fig. 7). Taken together, our results show that mutation of either, or both, ednrs results in reduced of soxE expression and skeletogenic NC differentiation, with the disruptions occurring most consistently in $\Delta e d n r a$ and $\Delta e d n r a+b$ individuals (Supplemental Tab. 2). Reductions in skeletogenic NC are also seen following edn1/ednra perturbation in model gnathostomes ${ }^{8,33,34}$, though disrupted soxE (sox9a) expression has only been reported in zebrafish ${ }^{33}$. We thus visualized sox9.S expression in $X$. laevis $\Delta$ ednra larvae, and found reduced expression (Fig. 20 and $\mathrm{p}$ ). This suggests regulation of soxE expression and NC skeletogenesis are deeply conserved functions of Edn signaling in vertebrates.

We next asked if $d l x$ genes are effectors of Edn signaling in the lamprey pharyngeal skeleton by comparing the phenotype of $\Delta e d n r$ and $\Delta d l x$ individuals. Mutation of $d l x A, d l x C$, and $d l x D$ alone, or in combination, resulted in disruptions of soxE2 and lecticanA expression similar to $\Delta$ ednr larvae (Fig. $2 \mathrm{H}$ and L, Supplemental Fig. 8A). At T30, $\Delta d l x$ individuals also had hypomorphic pharyngeal skeletons with gaps in the branchial basket, though they lacked the 
heart and pigment defects seen in $\Delta e d n r$ larvae (Supplemental Fig. 8B). The similarity of $\Delta e d n r$ and $\Delta d l x$ individuals suggest that Edn signaling works though $d l x$ genes in lamprey skeletogenic NC.

Like mouse ednra ${ }^{35}$, lamprey ednra transcripts mark presumptive cardiac mesoderm ${ }^{24}$ and lamprey $\Delta$ ednra larvae have severe heart defects (Supplemental Figs. 2, 3). We thus examined the expression of the FGFR homolog, fgfra, in $\triangle$ ednra larvae. In addition to being transcribed in lamprey cardiac mesoderm, functional studies suggest fgfra signaling is required for lamprey heart development ${ }^{22,36}$. We observed a strong reduction in cardiac fgfra expression in $\Delta$ ednra individuals (Fig. $2 \mathrm{M}$ and $\mathrm{N}$ ). This indicates that the heart edema seen in lamprey $\Delta e d n r a$ larvae is likely caused by reduced FGFR signaling in cardiac mesoderm.

Unlike ednra, ednrb is strongly expressed in trunk NC cells destined to form the dorsal root ganglia ${ }^{24}$. We thus examined the expression of several PNS markers ${ }^{37-40}$ in $\Delta e d n r b$ and $\Delta e d n r a+b$ embryos and larvae (Fig. 3A-M). Despite the reduced heads of $\Delta$ ednra $+b$ larvae, all major NC-derived PNS elements were present, including recently described SCPs ${ }^{38}$, though chromaffin-like cells near the pronephros were missing ${ }^{41}$ (Fig. 3A-C, arrowheads). This relatively mild defect is similar to gnathostome edn3/ednrb mutants and morphants which have only minor PNS deficiencies. These results suggest that development of the NC-derived PNS was largely Edn-independent in the last common ancestor of lamprey and gnathostomes.

In vitro binding assays ${ }^{42}$, and the similarity of edn and ednr mutant phenotypes ${ }^{2,33}$, suggest that Edn1 is the main ligand for Ednra, while Edn3 is the main ligand for Ednrb. To test if lamprey Ednra and Ednrb also have dedicated ligands, we mutated ednA, ednC, and ednE, the only edns expressed in tissue-specific patterns during lamprey development. Targeting ednC with three different sgRNAs yielded no reproducible mutant phenotype (see Methods). In contrast, lamprey $\Delta e d n A$ larvae displayed a combination of heart edema and skeletal defects that resembled hypomorphic $\Delta$ ednra individuals (though without ectopic pigmentation), while $\Delta e d n E$ larvae resembled $\Delta$ ednrb larvae (Fig $3 \mathrm{~N}-\mathrm{R}$, Supplemental Fig. 9). The incomplete loss of pigment in $\Delta e d n E / \Delta e d n r b$ larvae mimics gnathostome edn3/ednrb mutants ${ }^{43}$, though the variable phenotype could also reflect the mosaicism inherent in F0 mutagenesis. To address this, we created $\Delta e d n 3 X$. laevis. We found a high percentage had a complete loss of NCderived pigmentation (Supplemental Fig. 10, Supplemental Tab. 1), like edn3 mutant 
salamanders $^{44}$, confirming that F0 mutagenesis can generate near-null edn phenotypes a high frequencies. We conclude that all modern vertebrates have an edn dedicated to ednrb, and that amphibian NC-derived pigment cell development appears particularly dependent on edn3/ednrb signaling.

Inconclusive phylogenies ${ }^{17,24,45}$, similar expression patterns ${ }^{24}$, and similar mutant phenoptypes suggest that lamprey ednA and ednE could be cryptic orthologs of gnathostome edn1 and edn3, respectively. We thus used the recently completed sea lamprey germline genome ${ }^{23}$ to reevaluate ednr and edn phylogeny. Conserved synteny strongly confirms orthology of lamprey and gnathostome ednras and ednrbs ${ }^{24,46}$ (Supplemental Figs. 11,12). In contrast, phylogenetic analysis of flanking genes indicate that ednA and ednE are likely part of the edn2/4 and edn1/3 paralogy groups, respectively (Supplemental Figs. 11,12). Thus, while an Edn largely dedicated to Ednrb likely arose before the divergence of lamprey and gnathostomes, the lack of orthology between edn1 and ednA reveals a mainly Ednra-specific ligand evolved at least twice.

Work in invertebrate chordates suggests that the NC likely evolved from a population of migratory CNS cells that generated neurons and/or pigment cells ${ }^{47,48}$. In contrast, the NC of modern vertebrates is a patterned tissue made of many distinct subpopulations. Our work supports a model for the stepwise evolution of the major NC subpopulations (Fig. 4). We show here that lamprey larvae lacking all, or most, Edn signaling have a largely normal PNS, but an oropharyngeal skeleton reduced to unconnected cartilage bars. The similarity of this phenotype to the presumed vertebrate ancestor suggests Edn signaling evolved in an early stem vertebrate that had all major NC derivatives ${ }^{49-52}$. Because both ednrs are needed for normal oropharyngeal skeleton development in lamprey, and some gnathostomes express ednrb in skeletogenic $\mathrm{NC}^{24}$, we further posit the ancestral pre-duplication Ednr functioned in this derivative. After ednr duplication, but before the evolution of jaws, the subfunctionalization and specialization of ednra and ednrb contributed to the developmental divergence of the pigment and skeletogenic NC lineages. Finally, differences in the regulation of hand, and the role of edn3/ednrb in ENS development, suggest changes in Edn signaling targets may underlie divergence of the lamprey and gnathostome oropharyngeal skeletons and PNS. 


\section{Methods}

“F0” mutagenesis strategy. We used CRISPR/Cas9-mediated mutagenesis to induce deletions and insertions (indels) into the protein-coding exons of injected "F0" sea lamprey (Petromyzon marinus) and African clawed frog (Xenopus laevis) embryos as previously described $^{22,53-57}$. Though CRISPR/Cas9 is highly efficient in sea lamprey, differences in the efficiency of individual sgRNAs results in different ratios of wildtype and mutant alleles in F0 mosaic mutants. This variable mosaicism results in different sgRNAs producing phenotypically mutant individuals at different frequencies, with a range of severities. Previous work shows targeting an evolutionarily conserved, embryonically-expressed gene typically results in $20-90 \%$ of injected individuals displaying a gene-specific mutant phenotype ${ }^{22,53-56}$. Work in our lab with 35 guides targeting 20 different developmental regulators confirms this, with an average of $46 \%$ phenotypically mutant individuals produced per gene-specific sgRNA (Supplemental Tab. 1, Supplemental Fig. 13).

Also as previously reported, the severity of a CRISPR/Cas9-induced phenotype correlates well with the percentage mutant alleles; with most "severely affected" F0 mosaic mutants typically exhibiting 75\%-100\% mutant (indel) alleles ${ }^{22,53-56}$. Consistent with this, the 74 severely affected phenotypic mutants selected for genotyping in this study had an average of 88\% indel alleles at targeted loci (Supplemental Tab. 4). Importantly, every severely affected individual selected for genotyping had indel mutations at the targeted locus. Thus, as with traditional inbred mutant lines, the phenotype of CRISPR/Cas9-generated F0 mosaic mutants is a strong predictor of their genotype.

Based on these observations, we devised a strategy for creating, selecting, and analyzing CRISPR/Cas9-generated sea lamprey and $X$. laevis mutants. First, two or more unique sgRNAs were designed against protein-coding exons of the gene of interest. When possible, we selected unique, but evolutionarily conserved regions to increase the chances that in-frame deletions will disrupt functionally critical domains and yield loss-of-function alleles. Second, individual sgRNAs were co-injected with Cas9 protein or mRNA into zygotes or, in the case of $X$. laevis, zygotes and two-cell stage embryos. Third, F0 injected embryos were monitored daily and scored for morphological defects. Fourth, morphological defects associated with two or more sgRNAs targeting the same gene were designated as the "mutant phenotype" 
for that gene. For example, the unique pigmentation defect seen when targeting ednrb exons was deemed the "ednrb mutant phenotype" only after two different sgRNAs targeting the ednrb locus produced the same defect. Fifth, mutagenesis of the targeted loci was confirmed by genotyping several representative severely affected phenotypic mutants (see below for genotyping method). Sixth, once mutant genotype and mutant phenotype were linked by showing all selected mutants had mutant alleles, severely affected phenotypic mutants were picked for analyses via in situ hybridization, alcian blue staining, immunohistochemistry, and toluidine blue staining (see below for protocols). For $d l x$ sgRNAs, which resulted in unusually high mortality before larval stages, likely due to the early function of $d l x$ genes in neurectoderm patterning, severe phenotypic mutants were lightly fixed and genotyped after in situ hybridization analysis as recently described ${ }^{54,55}$. This additional step was performed to reconfirm the link between mutant phenotype and mutant genotype in the relatively small number of surviving $d l x$ mosaic mutants.

P. marinus sgRNA/Cas9 injections. We mutagenized the $P$. marinus $d l x A, d l x C, d l x D$, ednA, ednC, ednE, ednra, and ednrb loci by injecting zygotes with at least two unique sgRNAs per gene (Supplemental Tab. 1). To create ednra+b double mutants, zygotes were injected with four different combinations of the two most effective ednra and ednrb guides. $d / x A+C+D$ triple mutants were created using a single sgRNA $100 \%$ complementary to $d l x A$ and $d / x D$, with one mismatch to $d l x C$ (Supplemental Tab.1). As previously described, sgRNA target sites were chosen using all available transcriptome sequence data to avoid protein-coding off-targets ${ }^{22}$. Briefly, candidate sgRNA sequences demonstrating off-target matches with $>80 \%$ overall identity in the target site, and $>90 \%$ identity in the 3 ' half of the target site (closest to the PAM site) to any off-target sequence (with an NGG PAM site) were not used. Lamprey zygotes were injected as previously described with approximately $5 \mathrm{~nL}$ of a solution containing $400 \mathrm{pg}$ of sgRNA, and either $800 \mathrm{pg}$ of Cas9 protein (a 2:1 ratio of protein:sgRNA by mass) or $1 \mathrm{ng}$ of Cas9 mRNA, $5 \mathrm{mg} / \mathrm{mL}$ lysinated rhodamine dextran (LRD), and nuclease free water. For ednra + ednrb combined experiments, 200 pg of each of two sgRNAs were used with 800 pg of Cas 9 protein. Approximately 200-500 zygotes were injected per experiment, and each sgRNA/Cas9 
combination was injected into zygotes from at least two different pairs of wild caught sea lampreys.

As in other vertebrates ${ }^{58}$, microinjection of lamprey embryos causes increased mortality before gastrulation and developmental delay compared to uninjected sibling controls ${ }^{22}$. Due to differences in female health, person injecting, and progression of the spawning season, this microinjection-induced mortality can range from 10-90\%. However, after gastrulation, clutches of microinjected embryos have a survivorship to early larval stages (T26-T30) similar to uninjected siblings, typically around $90 \%$. This was true for all sgRNAs tested in this study, except for the $d l x$ sgRNAs, which had substantially increased mortality to larval stages compared to uninjected siblings, resulting in $30-40 \%$ survival to T26.5. We suspect this is due to the early roles of $d l x$ genes in neurectoderm patterning.

$X$. laevis sgRNA/Cas9 injections. Both the "Long" (-L) and "Short" (-S) homoeologs of $X$. laevis edn1, edn3, and ednra were simultaneously targeted ${ }^{57}$ (Supplemental Tab. 1). Zygotes or two-cell embryos were injected with a 5-10 nL droplet containing $800 \mathrm{pg}$ of a single sgRNA targeting both edn3.L (formerly edn3-a) and edn3.S (-b), or $400 \mathrm{pg}$ of sgRNAs targeting edn1 and ednra, and either $1 \mathrm{ng}$ of Cas9 mRNA, or $1.6 \mathrm{ng}$ of Cas9 protein. Approximately 50-200 zygotes were injected per experiment.

P. marinus CRISPR/Cas9 controls. To demonstrate that the phenotypes associated with each sgRNA injected were due to disruption of the targeted genes, rather than to off-targets, each $P$. marinus gene was targeted with at least two unique sgRNAs. All sgRNAs targeting the same gene produced the same mutant phenotype, though usually with different efficiencies (Supplemental Tab. 1).

To further validate sgRNA specificity in $P$. marinus, and to ensure that the CRISPR/Cas9 method does not artefactually cause any of the described defects, we used two negative control strategies. In addition to the negative control sgRNA described in our methods paper ${ }^{22}$ we tested an intron-spanning sgRNA partially complementary to two separate exons of the $P$. marinus ednrb gene (see Supplemental Tab. 1 for sequence). Neither sgRNA produced a phenotype (Supplemental Fig. 13), though both resulted in a slight developmental delay, as 
previously reported ${ }^{22}$. In addition to these "untargeted" sgRNA negative controls, we also injected more than 20 other sgRNAs complementary to the exons other $P$. marinus developmental genes (Supplemental Fig. 13). These sgRNAs were designed to disrupt developmental regulators expressed in the developing head at the same time as ednr, edn, and $d l x$. None of these negative control sgRNAs yielded the ednr or edn mutant phenotypes, though three sgRNAs (a2cg1, p19g1, and w11g3) produced phenotypes grossly similar to dlx mutants (Supplemental Fig. 13).

Severe heart edema (approximate heart volume greater than $3 x$ normal by visual inspection) is part of both the ednra and ednA mutant phenotypes, and occurs at a high frequency in embryos injected with sgRNAs targeting fgf8/17/18 22 (Supplemental Fig. 13). This raised the possibility that heart edema could be a non-specific side-effect of sgRNA/Cas9 injection. To test this, we counted the number of negative control larvae, aside from those injected with fgf8/17/18 sgRNA, displaying heart edema (Supplemental Fig. 13). Of 21 pools of larvae injected with 21 different negative control sgRNAs, 9 pools displayed no detectable heart edema, while 11 displayed heart edema of various severities at a frequency of $7.7 \%$ or lower. 1 sgRNA yielded severe heart edema at a frequency of $27 \%$. These data show that severe heart edema is not a general side-effect of the CRISPR/Cas9 method in lamprey.

X. laevis CRISPR/Cas9 controls. An edn3 morphant phenotype was previously reported in $X$. laevis $^{59}$. An sgRNA designed to simultaneously target the edn3.L and edn3.S homoeologs yielded a severe version of the $X$. laevis edn 3 morphant phenotype that mimicked salamander edn3 mutants ${ }^{44}$, confirming its specificity. For edn1 and ednra, we designed separate sgRNAs against the $L$ and $S$ homoeologs and performed negative controls by individually injecting each sgRNA separately as reported previously ${ }^{57}$. This strategy relies on redundancy of the $X$. laevis homoeologs to show that neither sgRNA alone causes any spurious morphological defects. The fact that defects are only obtained by simultaneous disruption of homoeologs, serves as a control showing that the phenotype is specifically due to a loss of edn1 and ednra function ${ }^{57}$.

P. marinus husbandry. $P$. marinus fertilizations and husbandry were carried out as described previously ${ }^{22}$. Adult spawning phase sea lampreys were housed in $200 \mathrm{~L}$ tanks containing reverse 
osmosis purified water with $800-1000$ ppm artificial sea salt. Water in the tanks was completely replaced daily. Once ripe, the animals were stripped of gametes into Pyrex dishes, where in vitro fertilization took place in deionized water containing 400-600 ppm artificial sea salt. All animals were wild-caught from fresh water streams during their late spring/early summer spawning season, with the majority being derived from an invasive population in Lake Huron. A small fraction (1\%), were trapped at the Holyoke Dam in Massachusetts. Each sgRNA was injected into clutches from at least of two different pairs of adults. Embryos and larvae were kept at $18^{\circ} \mathrm{C}$ in Pyrex dishes containing deionized water and $400-600$ ppm artificial sea salt. Depending on female health and time of year, uninjected sea lamprey embryos display survivorship to st. T26.5 from 1\%-99\%. Dead embryos and larvae were removed daily from each dish, and the water was changed at least every other day. All $P$. marinus staging was according to Tahara $^{60}$. All P. marinus husbandry and experiments were in accordance with CUBoulder IACUC protocol \#2392.

$X$. laevis husbandry. $X$. laevis fertilizations and husbandry were performed according to standard methods ${ }^{61}$. Adult females were induced to ovulate via injection of Human Chorionic Gonadotropin (HCG), and eggs were stripped into petri dishes. Testes were dissected from males, homogenized, and applied to the eggs for in vito fertilization. All frog staging was according to Nieuwkoop \& Faber ${ }^{61}$. All $X$. laevis husbandry and experiments were in accordance with CU-Boulder IACUC protocol \#2392.

Scoring of mutant phenotypes. Successfully injected embryos were identified by fluorescence of the LRD lineage tracer at 4-6 days post fertilization and dead and LRD-negative larvae were discarded. Successfully injected embryos and larvae were then monitored for morphological abnormalities as they developed. Suites of morphological defects associated with injection of a particular sgRNA, and also seen when injecting one or more other sgRNAs targeting the same gene, were designated as the "mutant phenotype" for that gene. Of embryos and larvae displaying the "mutant phenotype", we deduced, based on previous work, that most severe had more than $75 \%$ mutant alleles and were likely near null-mutants ${ }^{22,53-56}$. This was assumption was supported by genotyping representative severe mutants for all targeted genes (see 
"Genotyping" section below)

For each gene, we focused on "severely affected" mutants for detailed morphological and histological analyses. The severe mutant phenotype of all genes was apparent at pharyngula stages onward (st. T26.5 for lamprey, st. 41 for $X$. laevis) and defined as follows. For $X$. laevis $\Delta e d n 1$ and $\Delta e d n r a$ the severe mutant phenotype was defined as a reduction in head size (all structures anterior to the heart) to approximately $70 \%$ of WT size or smaller. For $P$. marinus $\triangle e d n A$ the severe mutant phenotype was defined as a reduction in head size to approximately $70 \%$ of its WT size or smaller, together with heart edema. For $\Delta$ ednra, the severe mutant phenotype was defined as a reduction in head size to approximately $70 \%$ of its WT size or smaller, together with heart edema, and ectopic pigmentation around the heart. For $\Delta d l x A$, $\Delta d l x C$, and $\Delta d l x D$, severe mutants were defined as having a head reduced to approximately $70 \%$ of WT size or smaller. For $\Delta e d n 3, \Delta e d n E$, and $\Delta e d n r b$ severe mutants were defined as having a $50 \%$ reduction in the number of melanophores or greater (in the case of $X$. laevis injected unilaterally at the 2 cell stage, this applies only to the injected side). For the $\Delta e d n r a+b$, the severe mutant phenotype was defined as an approximately $70 \%$ reduction in head size, heart edema, and approximately 50\% reduced pigmentation. All larvae demonstrating a "severe mutant phenotype" were counted and are presented as fraction of the total number of LRDpositive embryos and larvae that survived to fixation at a stage were phenotype could be scored (Supplemental Tab. 1, 2).

As in other vertebrates ${ }^{58}$, sea lamprey embryos injected with negative control sgRNAs, DNA constructs, or any other synthetic oligonucleotide, display a slight developmental delay. In sea lamprey we find that a delay of $\sim 5 \%$ is typical, i.e. 10 day old injected embryos and larvae typically appear 9.5 days old compared to unmanipulated siblings. Thus, developmental events such as somite segregation, yolk absorption, gill openings, and melanin deposition ${ }^{60}$ were used, rather than days post-fertilization, to stage-match mutant and negative control embryos.

Genotyping. To confirm successful mutagenesis, individual severe mutants were genotyped by preparing genomic DNA, PCR amplifying the target site, subcloning the amplicons, and Sanger sequencing individual alleles as previously described ${ }^{22,53-56}$. Target sites and genotyping primers for each sgRNA are in Supplemental Tab. 1. We genotyped at least 3 severely affected 
individuals for each targeted gene or combination of genes (Supplemental Fig. 2, 5, 6, 8-10, Supplemental Tab. 2) except in the case of $P$. marinus ednrb sgRNA2, which likely lies immediately adjacent to an intron/exon boundary conserved across jawed vertebrates (on the 5' end of exon 4 in zebrafish ednraa [NM_001099445.2]), and is incompletely assembled in all three publicly available genomic assemblies (including the 2017 petMar3 ${ }^{23}$ ). For $d l x$ mutants, genotyping after histological analysis of lightly fixed mutants was performed as previously described $^{54-56}$.

Frequently, we found six or more unique indel alleles at a given locus in a single specimen (in $X$. laevis, we consider the homoeologous " $L$ " and " $S$ " loci separately), which indicates that biallelic Cas9-driven mutagenesis is still occurring after the second cleavage event in both species. As previously reported ${ }^{22,57,62}$, when insertions of DNA fragments were discovered, these motifs often appeared on reverse or forward strand very close to the target site/lesion (see green and purple nucleotides in Supplemental Fig. 2, 5, 6, 8-10).

\section{Histological staining, In situ hybridization, and immunohistochemistry. All in situ}

hybridization (ISH), alcian blue cartilage staining, and toluidine blue staining was carried out as described previously ${ }^{15,63,64}$. Neurofilament immunohistochemistry (IHC) was as described previously ${ }^{39}$, with the addition of $1 \%$ dimethyl sulfoxide (DMSO) to the phosphate buffer solution prior to the blocking step. To ensure equivalent signal development in injected and WT individuals, morphologically stage-matched WT embryos and larvae were included in every ISH, $\mathrm{IHC}$, and histological staining experiment, with WT and treated larvae kept in same tubes, with the caudal $1 / 4$ cut-off for identification when necessary. In addition, to verify that none of the disrupted expression patterns or aberrant histology of mutants could be explained by slight developmental delay, a known side-effect of microinjection, WT embryos one stage younger were also used for comparisons, i.e. morphological st. T26.5 mutant larvae were compared to both morphological st. T25.5 and st. T26.5 wildtype larvae. The number of embryos and larvae processed for each histological method, and the frequencies of aberrations, are reported in Supplemental Tab. 2. 
Statistical analyses. We have never observed the ednra, ednrb, ednA, and ednE mutant phenotypes in wildtype or negative control embryos. In other words, the ednra, ednrb, ednA, and ednE phenotypes are only seen in embryos and larvae injected with Cas9 and sgRNAs targeting these genes. Similarly, we have never observed the reduced expression patterns we report in wildtype or negative control embryos. However, non-specific body axis deformities (mainly incomplete yolk-sac extension) occur at a frequency of $5-8 \%$ in surviving uninjected, and negative control-injected larvae. While these deformities are qualitatively different from the ednra, ednrb, ednA, and ednE mutant phenotypes, we used this background level of developmental deformity as a proxy to estimate mutant phenotype frequencies in negative control sea lamprey larvae (Supplemental Tab. 3). Using the conservative estimate that one out of ten negative control (untreated) individuals will spontaneously display the observed phenotypes, we applied Fisher's exact test to evaluate the null hypothesis that our treatments can be explained by a high 'background' level of developmental deformities. This hypothesis is rejected with $P$ values of $<.02$ for all mutant phenotypes, with the majority having $P$ values $<.0001$ (Supplemental Tab. 3).

Most in situ hybridization (ISH) assays were performed on embryos and larvae displaying the severe morphological phenotype. Because these specimens were non-randomly selected phenotypic mutants, statistical analysis is inappropriate. For pre-selected phenotypic mutants, we report the fraction of those assayed by ISH displaying disrupted gene expression patterns in Supplemental Tab. 3. The remaining ISH assays were performed on embryos before the mutant phenotype became apparent and severe mutants could be selected. In these cases, selected individuals were a random sample of the pool of sgRNA/Cas9 individuals and could be compared to untreated controls with Fisher's exact test (Supplemental Tab. 3). For these experiments, we assumed spontaneous disruption of gene expression in 5 out of 100 of untreated, wildtype embryos and larvae. We view this assumption as conservative as we have never observed variation in gene expression patterns in wildtype embryos that have been properly processed for in situ hybridization or immunohistochemistry. Under this assumption, every reported effect of "no expression change" in this work is consistent with a null hypothesis of no effect $/ 5 \%$ background levels of gene disruption (Fisher's exact test $P>0.35$ ). For all genes 
we report as having discontiguous, missing, or otherwise reduced gene expression after treatment, the null hypothesis is rejected with $\mathrm{P}<<.0001$ (Supplemental Tab. 3).

Synteny and phylogenetic analysis. For the Endothelin receptors and ligands, we looked at synteny at each locus where possible (Supplemental Fig.11,12). The synteny analysis was performed by finding the coding sequences in the $2018 P$. marinus genome ${ }^{23}$ via the UCSC genome browser (http://genome.ucsc.edu/) and comparing the neighboring genes to that of chicken (Gallus gallus) and/or human (Homo sapiens) as published previously ${ }^{46}$. For the Endothelin ligands, synteny information alone was ambiguous and amino acid similarity across large phylogenetic distances is poor, so we relied on the relatedness of the closely linked hivep and phactr gene products to deduce the likely evolutionary history of these gene families ${ }^{45}$ (Supplemental Fig. 12). For Ednrs, we repeated an amino acid similarity analysis according to the same methods as we used previously ${ }^{24}$, but with a subset of sequences. All phylogenetic and molecular evolutionary analyses were conducted using MEGA version $6^{65}$. See Supplemental Tab. 5. for all accession numbers associated with these analyses. 


\section{Data Availability}

All data generated or analyzed, and all methods used during this study are summarized in this published article (and its Supplemental information files). The raw data and images are available from the first and second authors upon reasonable request.

\section{Acknowledgements}

The authors thank Scott Miehls at the USGS Hammond Bay Biological Station and Brittney Laflamme at the Holyoke Dam for providing adult sea lampreys, Bilge Birsoy, Jianli Shi, and Michael Klymkowsky for assistance with $X$. laevis fertilizations, Zachary Root for assistance with $X$. laevis and sea lamprey injections and husbandry, Claire Altier for assistance with juvenile $X$. laevis husbandry, and Shane Schwikert for pro bono statistics consultation. DMM, TAS, DJ, MR, JLM, and MVC were supported by National Science Foundation grants IOS 1656843, IOS 1257040, and IOS 0920751 to DMM. HPS and AWH were supported by the University of Colorado, Boulder Undergraduate Research Opportunities Program. DJ was supported by the European Union's Horizon 2020 research and innovation programme under the Marie Skłodowska-Curie grant agreement No 751066 and by the Scientific Grant Agency of the Slovak Republic VEGA grant No.1/0415/17.

\section{Author Contributions}

D.M.M. conceived the project. T.S., D.J., M.R., and D.M.M. designed the experiments. All authors performed experiments and collected data. D.M.M. and T.S. wrote the manuscript. All authors discussed and provided input on the final manuscript.

\section{Author Information}

- The authors declare no competing interests

- Correspondence and requests for materials should be addressed to daniel.medeiros@colorado.edu 


\section{Figure Legends}

Figure 1 | Lamprey $\Delta$ ednr larvae have pharyngeal skeleton defects, and reduced intermediate domain dIx expression. A, Illustration of the larval sea lamprey pharyngeal skeleton at st. T30. B-G, Toluidine blue-stained sagittal section of the oral mucocartilage (B-D) and flat-mounted alcian blue stain of the branchial basket (E-G) at. st. T30 in wildtype (B,E) $\Delta e d n r a(\mathrm{C}, \mathrm{F})$ and $\Delta e d n r a+b(\mathrm{D}, \mathrm{G})$ larvae. $\Delta e d n r a$ and $\Delta e d n r a+b$ mutants have reduced pharyngeal skeletons with gaps and missing elements (arrows). $\mathbf{H}$, Illustration of $d l x$ and hand expression in the lamprey head at T26.5 I-Q, $d l x A, d l x D$, and hand expression in WT $(\mathrm{I}, \mathrm{L}, \mathrm{O})$, $\Delta e d n r a(\mathrm{~J}, \mathrm{M}, \mathrm{P})$, and $\Delta e d n r a+b(\mathrm{~K}, \mathrm{~N}, \mathrm{Q})$ mutants. Disruptions in the differentiated pharyngeal skeleton at. st. T30, and intermediate domain $d l x$ expression at st. T26.5 are seen in both $\Delta e d n r a$ and $\Delta e d n r a+b$ larvae, but are most pronounced in $\Delta e d n r a+b$ individuals. In contrast, ventral hand expression persists in $\Delta e d n r a$ and $\Delta e d n r a+b$ larvae. Asterisks indicate pharyngeal arches with missing expression. All scale bars indicate $100 \mu \mathrm{m}$. Scale bar in B applies to B,C,D. Scale bar in E applies to E,F,G. Scale bar in I applies to I-Q. All panels show left lateral views. See Supplemental Tab. 1-4 for sgRNA sequences, genotyping data, numbers affected, and statistical analyses.

Figure 2 | Skeletogenic NC development is disrupted in lamprey $\Delta e d n r$ and $\Delta d I x$, and in $X$. laevis $\Delta$ ednra larvae, and cardiac mesoderm development is disrupted in lamprey $\Delta$ ednra larvae. A-B, Expression of twist $A$ in migratory NC at st. T23 in WT (A) and $\Delta e d n r a+b$ larvae (B). C-D, Expression of myc in post-migratory NC at st. T26.5 in WT (C) and $\Delta e d n r a+b$ larvae (D). The expression of twistA, myc, and other general cranial NC markers (Supplemental Fig. 7), suggests that cranial NC formation is largely normal in $\Delta$ ednr larvae. E-L, reduced and discontiguous expression of soxE2 (E-H) and lecticanA (I-L), in WT (E,I), Dednra (F,J), $\Delta e d n r a+b(\mathrm{G}, \mathrm{K})$, and $\Delta d l x A(\mathrm{H}, \mathrm{L})$ larvae at st. T26.5. $\Delta e d n r a, \Delta e d n r b$, and $\Delta d l x$ mutants have similar disruptions in soxE2 and lecticanA expression, showing skeletogenic NC development is disrupted when these genes are mutated. M-N, FGFRa expression in cardiac mesoderm (M) is reduced in $\Delta$ ednra mutants (N). O-P, Like lamprey $\Delta$ ednra and $\Delta$ ednrb larvae, Xenopus laevis $\Delta$ ednra larvae $(\mathrm{N})$ have disruptions in soxE (sox9.s) expression in post-migratory NC (st. N.F. 33/34). All panels show left lateral views. All scale bars indicate $100 \mu \mathrm{m}$. Scale bar in A applies 
to A-D. Scale bar in E applies to E-L. Scale bar in M applies to M-N. Scale bar in O applies to OP. See Supplemental Tab. 1-4 for sgRNA sequences, genotyping data, numbers affected, and statistical analyses.

Figure 3 | Most PNS elements form normally in lamprey $\Delta e d n r$ larvae, and $\Delta e d n$ and $\Delta$ ednr larvae have similar phenotypes. A-C, Neurofilament immunohistochemistry at st. T27 reveals all major facial nerves (white arrows) in WT (A), $\Delta$ ednrb (B), and $\Delta$ ednra $+b$ mutants (C), though a population of Neurofilament-positive cells near the pronephros is missing (arrowheads B,C). D-G, phox2 expression at st. 26.5 reveals forming epibranchial ganglia (white arrows) and the recently described "Schwann cell precursors" (black arrows) in WT (D), $\Delta e d n r a(E), \Delta e d n r b$ $(\mathrm{F})$, and $\Delta e d n r a+b$ mutants $\mathrm{G})$. Despite reduced heads, $\Delta$ ednr mutants have largely normal epibranchial ganglia. H-M, Comparisons of foxD-A $(\mathrm{H}, \mathrm{I})$, soxE2 $(\mathrm{J}, \mathrm{K})$, and $\operatorname{soxB1b}(\mathrm{L}, \mathrm{M})$ expression in WT $(\mathrm{H}, \mathrm{J}, \mathrm{L})$ and $\Delta$ ednra+b mutants $(\mathrm{I}, \mathrm{K}, \mathrm{M})$ at st. 26.5 suggest the dorsal root ganglia (white arrowheads) form normally in $\Delta e d n r a, \Delta e d n r b$, and $\Delta e d n r a+b$ mutants. N, WT st. T30 larvae, O-R, $\Delta$ edn mutants phenocopy mild $\Delta e d n r$ mutants. $\Delta$ ednA mutants $(\mathrm{O})$ recapitulate the hypomorphic head and heart edema (brackets) of $\Delta$ ednra mutants $(P)$, but lack the ectopic pigmentation caused by ednra disruption (arrowheads). $\Delta$ ednE mutants (Q) have reduced pigmentation, resembling $\Delta$ ednrb mutants (R). All scale bars indicate $100 \mu \mathrm{m}$. Scale bar in A applies to A-C. Scale bar in D applies to D-G. Scale bar in H applies to H-I. Scale bar in J applies to J-M. Scale bar in $\mathrm{N}$ applies to O-S. All panels show left lateral views, save J-M which are dorsal views of the trunk (anterior to left). See Supplemental Tab. 1-4 for sgRNA sequences, genotyping data, numbers affected, and statistical analyses.

\section{Figure 4 | The stepwise evolution of Edn signaling in neural crest development deduced} from lamprey and gnathostome Edn/Ednr mutant phenotypes. A, The similarity of Edn signaling-deficient lamprey larvae to basal vertebrate fossils, and the finding that Edn signaling is dispensable for early NC development in all modern vertebrates, suggest the vertebrate ancestor had a fully potent NC that developed in the absence of Edn signaling. B, The large reductions in NC-derived skeletal tissue and pigment cells in lamprey and gnathostome $\Delta e d n r a+b$ mutants, but not NC-derived peripheral neurons, suggest the recruitment of 
Endothelin signaling contributed to the expansion of skeletal, and other non-neural NC derivatives, in stem vertebrates. C, Subsequent duplication and specialization of the ednra and ednrb signaling pathways resulted in three NC subpopulations with different Endothelin signaling requirements. Differences in the phenotypes of lamprey and gnathostome Edn signaling mutants suggest the roles of Edn signaling in pharyngeal skeleton patterning has diverged in jawed and jawless vertebrates. D, In the sea lamprey, Ednrb works with Ednra in skeletogenic NC, while hand transcription in the ventral pharynx is Endothelin signalingindependent. D', In modern gnathostomes, pharyngeal skeleton development is Ednrb-signaling independent, and hand expression in the ventral pharynx requires Ednra signals. E, The deduced character states in A-C mapped onto a phylogenetic tree of extant chordate groups. 


\section{References}

1 Simoes-Costa, M. \& Bronner, M. E. Establishing neural crest identity: a gene regulatory recipe. Development 142, 242-257, doi:10.1242/dev.105445 (2015).

2 Miller, C. T., Schilling, T. F., Lee, K., Parker, J. \& Kimmel, C. B. sucker encodes a zebrafish Endothelin-1 required for ventral pharyngeal arch development. Development 127, 3815-3828 (2000).

3 Miller, C. T., Yelon, D., Stainier, D. Y. \& Kimmel, C. B. Two endothelin 1 effectors, hand2 and bapx1, pattern ventral pharyngeal cartilage and the jaw joint. Development 130, 1353-1365 (2003).

$4 \quad$ Krauss, J. et al. Endothelin signalling in iridophore development and stripe pattern formation of zebrafish. Biol Open 3, 503-509, doi:10.1242/bio.20148441 (2014).

5 Baynash, A. G. et al. Interaction of endothelin-3 with endothelin-B receptor is essential for development of epidermal melanocytes and enteric neurons. Cell 79, 1277-1285 (1994).

6 Stock, D. W. \& Whitt, G. S. Evidence from 18 S ribosomal RNA sequences that lampreys and hagfishes form a natural group. Science 257, 787-789 (1992).

7 Miller, C. T. \& Kimmel, C. B. Morpholino phenocopies of endothelin 1 (sucker) and other anterior arch class mutations. Genesis 30, 186-187, doi:10.1002/gene.1061 [pii] (2001).

8 Clouthier, D. E. et al. Cranial and cardiac neural crest defects in endothelin-A receptor-deficient mice. Development 125, 813-824 (1998).

9 Ruest, L. B., Xiang, X., Lim, K. C., Levi, G. \& Clouthier, D. E. Endothelin-A receptor-dependent and independent signaling pathways in establishing mandibular identity. Development 131, 4413-4423, doi:10.1242/dev.01291dev.01291 [pii] (2004).

Tavares, A. L. P. et al. Ectodermal-derived Endothelin1 is required for patterning the distal and intermediate domains of the mouse mandibular arch. Developmental Biology 371, 47-56, doi:Doi 10.1016/J.Ydbio.2012.08.003 (2012). Charite, J. et al. Role of DIx6 in regulation of an endothelin-1-dependent, dHAND branchial arch enhancer. Genes Dev 15, 3039-3049, doi:10.1101/gad.931701 (2001).

12 Lee, H. O., Levorse, J. M. \& Shin, M. K. The endothelin receptor-B is required for the migration of neural crest-derived melanocyte and enteric neuron precursors. Developmental Biology 259, 162-175, doi:10.1016/S0012-1606(03)00160-X (2003).

13 Parichy, D. M. et al. Mutational analysis of endothelin receptor b1 (rose) during neural crest and pigment pattern development in the zebrafish Danio rerio. Developmental Biology (Orlando) 227, 294-306, doi:10.1006/dbio.2000.9899S0012-1606(00)99899-3 [pii] (2000).

14 Hosoda, K. et al. Targeted and natural (piebald-lethal) mutations of endothelin-B receptor gene produce megacolon associated with spotted coat color in mice. Cell 79, 1267-1276 (1994).

15 Cerny, R. et al. Evidence for the prepattern/cooption model of vertebrate jaw evolution. Proceedings of the National Academy of Sciences of the United States of America 107, 17262-17267, doi:1009304107 [pii]10.1073/pnas.1009304107 (2010). Langeland, J. A., Neidert, A. H., Virupannavar, V. \& Hooker, G. W. Lamprey Dlx genes and early vertebrate evolution. Proceedings of the National Academy of Sciences of the United States of America 98, 1665-1670 (2001). mechanisms involving Dlx and endothelins in vertebrates. Developmental Biology (Orlando), doi:S00121606(10)00098-9 [pii]10.1016/j.ydbio.2010.02.013 (2010). Johnels, A. G. On the development and morphology of the skeleton of the head of Petromyzon. . Acta Zoologica (Stockholm) 29, 140-277 (1948). Gaskell, W. H. The Origin of Vertebrates. (Longmans, Green, and Co., 1908). Damas, $\mathrm{H}$. Recherches sur la de velopment de Lampetra fluviatilis L. Contribution a' l'e tude de la céphalogene'se des verte'bre' s. Arch. Biol. 55, 5-284 (1944). Kuratani, S. Evolution of the vertebrate jaw from developmental perspectives. Evolution \& Development 14, 76-92, doi:10.1111/j.1525-142X.2011.00523.x (2012). Square, T. et al. CRISPR/Cas9-mediated mutagenesis in the sea lamprey Petromyzon marinus: a powerful tool for understanding ancestral gene functions in vertebrates. Development 142, 4180-4187, doi:10.1242/dev.125609 (2015). Smith, J. J. et al. The sea lamprey germline genome provides insights into programmed genome rearrangement and vertebrate evolution. Nat Genet 50, 270-277, doi:10.1038/s41588-017-0036-1 (2018). Square, T., Jandzik, D., Cattell, M., Hansen, A. \& Medeiros, D. M. Embryonic expression of endothelins and their receptors in lamprey and frog reveals stem vertebrate origins of complex Endothelin signaling. Sci Rep 6, 34282, doi:10.1038/srep34282 (2016). 
Wada, H., Yao, T., Ohtani, K. \& Kuratani, S. Development of Lamprey Mucocartilage and its Dorsal-Ventral Patterning by Endothelin Signaling, With Insight Into Vertebrate Jaw Evolution. Journal of Experimental Zoology Part B-Molecular and Developmental Evolution 316B, 339-346, doi:Doi 10.1002/Jez.B.21406 (2011). Clouthier, D. E., Garcia, E. \& Schilling, T. F. Regulation of Facial Morphogenesis by Endothelin Signaling: Insights From Mice and Fish. Am J Med Genet A 152A, 2962-2973, doi:Doi 10.1002/Ajmg.A.33568 (2010). Fujimoto, S., Oisi, Y., Kuraku, S., Ota, K. G. \& Kuratani, S. Non-parsimonious evolution of hagfish DIx genes. BMC Evol Biol 13, 15, doi:1471-2148-13-15 [pii]10.1186/1471-2148-13-15 (2013). Sauka-Spengler, T., Meulemans, D., Jones, M. \& Bronner-Fraser, M. Ancient evolutionary origin of the neural crest gene regulatory network. Developmental Cell 13, 405-420, doi:Doi 10.1016/J.Devcel.2007.08.005 (2007). Lakiza, O., Miller, S., Bunce, A., Lee, E. M. J. \& McCauley, D. W. SoxE gene duplication and development of the lamprey branchial skeleton: Insights into development and evolution of the neural crest. Developmental Biology 359, 149-161, doi:Doi 10.1016/J.Ydbio.2011.08.012 (2011). Meulemans, D., McCauley, D. \& Bronner-Fraser, M. Id expression in amphioxus and lamprey highlights the role of gene cooption during neural crest evolution. Dev Biol 264, 430-442 (2003). McCauley, D. W. \& Bronner-Fraser, M. Importance of SoxE in neural crest development and the evolution of the pharynx. Nature 441, 750-752 (2006). Nair, S., Li, W., Cornell, R. \& Schilling, T. F. Requirements for Endothelin type-A receptors and Endothelin-1 signaling in the facial ectoderm for the patterning of skeletogenic neural crest cells in zebrafish. Development 134, 335-345, doi:dev.02704 [pii]10.1242/dev.02704 (2007). Bonano, M. et al. A new role for the Endothelin-1/Endothelin-A receptor signaling during early neural crest specification. Developmental Biology (Orlando) 323, 114-129, doi:S0012-1606(08)01117-2 [pii]10.1016/j.ydbio.2008.08.007 (2008). Asai, R. et al. Endothelin receptor type A expression defines a distinct cardiac subdomain within the heart field and is later implicated in chamber myocardium formation. Development 137, 3823-3833, doi:10.1242/dev.054015 (2010). Jandzik, D. et al. Roles for FGF in lamprey pharyngeal pouch formation and skeletogenesis highlight ancestral functions in the vertebrate head. Development 141, 629-638, doi:141/3/629 [pii]10.1242/dev.097261 (2014). Haming, D. et al. Expression of Sympathetic Nervous System Genes in Lamprey Suggests Their Recruitment for Specification of a New Vertebrate Feature. PLoS ONE 6, doi:ARTN e26543DOI 10.1371/journal.pone.0026543 (2011). Green, S. A., Uy, B. R. \& Bronner, M. E. Ancient evolutionary origin of vertebrate enteric neurons from trunkderived neural crest. Nature 544, 88-91, doi:10.1038/nature21679 (2017). McCauley, D. W. \& Bronner-Fraser, M. Conservation of Pax gene expression in ectodermal placodes of the lamprey. Gene 287, 129-139 (2002). Cattell, M. V., Garnett, A. T., Klymkowsky, M. W. \& Medeiros, D. M. A maternally established SoxB1/SoxF axis is a conserved feature of chordate germ layer patterning. Evolution \& Development 14, 104-115, doi:Doi 10.1111/J.1525-142x.2011.00525.X (2012). Higashiyama, H. et al. On the Vagal Cardiac Nerves, with Special Reference to the Early Evolution of the Head-Trunk Interface. J Morphol 277, 1146-1158, doi:10.1002/jmor.20563 (2016). Karne, S., Jayawickreme, C. K. \& Lerner, M. R. Cloning and Characterization of an Endothelin-3 Specific Receptor (Et(C) Receptor) from Xenopus-Laevis Dermal Melanophores. Journal of Biological Chemistry 268, 19126-19133 (1993). Reissmann, M. \& Ludwig, A. Pleiotropic effects of coat colour-associated mutations in humans, mice and other mammals. Semin Cell Dev Biol 24, 576-586, doi:10.1016/j.semcdb.2013.03.014 (2013). Woodcock, M. R. et al. Identification of Mutant Genes and Introgressed Tiger Salamander DNA in the Laboratory Axolotl, Ambystoma mexicanum. Sci Rep 7, 6, doi:10.1038/s41598-017-00059-1 (2017). Braasch, I., Volff, J. N. \& Schartl, M. The endothelin system: evolution of vertebrate-specific ligand-receptor interactions by three rounds of genome duplication. Molecular Biology \& Evolution 26, 783-799, doi:msp015 [pii]10.1093/molbev/msp015 (2009). Braasch, I. \& Schartl, M. Evolution of endot
doi:10.1016/j.ygcen.2014.06.028 (2014). Medeiros, D. M. The evolution of the neural crest: new perspectives from lamprey and invertebrate neural crest-like cells. Wiley Interdisciplinary Reviews: Developmental Biology, n/a-n/a, doi:10.1002/wdev.85 (2012). Abitua, P. B., Wagner, E., Navarrete, I. A. \& Levine, M. Identification of a rudimentary neural crest in a nonvertebrate chordate. Nature 492, 104-107, doi:nature11589 [pii]10.1038/nature11589 (2012). Mallatt, J. \& Chen, J. Y. Fossil sister group of craniates: Predicted and found. J Morphol 258, 1-31 (2003). Morris, S. C. \& Caron, J. B. A primitive fish from the Cambrian of North America. Nature 512, 419-U413, doi:Doi 10.1038/Nature13414 (2014). 
51 Shu, D. et al. A new species of yunnanozoan with implications for deuterostome evolution. Science 299, 1380-1384 (2003).

52 Shu, D. G. et al. Head and backbone of the Early Cambrian vertebrate Haikouichthys. Nature 421, 526-529 (2003).

53 Zu, Y. et al. Biallelic editing of a lamprey genome using the CRISPR/Cas9 system. Sci Rep 6, 23496, doi:10.1038/srep23496 (2016).

54 York, J. R., Yuan, T., Lakiza, O. \& McCauley, D. W. An ancestral role for Semaphorin3F-Neuropilin signaling in patterning neural crest within the new vertebrate head. Development 145, doi:10.1242/dev.164780 (2018).

55 York, J. R., Yuan, T., Zehnder, K. \& McCauley, D. W. Lamprey neural crest migration is Snail-dependent and occurs without a differential shift in cadherin expression. Developmental Biology 428, 176-187, doi:10.1016/j.ydbio.2017.06.002 (2017).

56 Yuan, T., York, J. R. \& McCauley, D. W. Gliogenesis in lampreys shares gene regulatory interactions with oligodendrocyte development in jawed vertebrates. Dev Bio/ 441, 176-190, doi:10.1016/j.ydbio.2018.07.002 (2018).

57 Wang, F. Q. et al. Targeted gene disruption in Xenopus laevis using CRISPR/Cas9. Cell Biosci 5, doi:ARTN 1510.1186/s13578-015-0006-1 (2015).

58 Rosen, J. N., Sweeney, M. F. \& Mably, J. D. Microinjection of zebrafish embryos to analyze gene function. $J$ Vis Exp, doi:10.3791/1115 (2009).

59 Kawasaki-Nishihara, A., Nishihara, D., Nakamura, H. \& Yamamoto, H. ET3/Ednrb2 Signaling Is Critically Involved in Regulating Melanophore Migration in Xenopus. Developmental Dynamics 240, 1454-1466, doi:Doi 10.1002/Dvdy.22649 (2011).

60 Tahara, Y. Normal stages of development in the lamprey Lampetra reissneri (Dybowski). Zoolog Sci 5, 109118 (1988).

61 Sive, H. L., Grainger, R. M. \& Harland, R. M. Early development of Xenopus laevis : a laboratory manual. (Cold Spring Harbor Laboratory Press, 2000).

62 Flowers, G. P., Timberlake, A. T., Mclean, K. C., Monaghan, J. R. \& Crews, C. M. Highly efficient targeted mutagenesis in axolotl using Cas9 RNA-guided nuclease. Development 141, 2165-2171, doi:Doi 10.1242/Dev.105072 (2014).

63 Square, T. et al. A gene expression map of the larval Xenopus laevis head reveals developmental changes underlying the evolution of new skeletal elements. Developmental Biology 397, 293-304, doi:Doi 10.1016/J.Ydbio.2014.10.016 (2015).

64 Aigler, S. R., Jandzik, D., Hatta, K., Uesugi, K. \& Stock, D. W. Selection and constraint underlie irreversibility of tooth loss in cypriniform fishes. Proceedings of the National Academy of Sciences of the United States of America 111, 7707-7712, doi:10.1073/pnas.1321171111 (2014).

65 Tamura, K., Stecher, G., Peterson, D., Filipski, A. \& Kumar, S. MEGA6: Molecular Evolutionary Genetics Analysis Version 6.0. Mol Biol Evol 30, 2725-2729, doi:Doi 10.1093/Molbev/Mst197 (2013). 


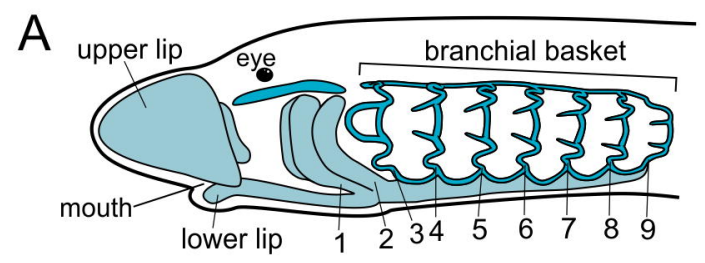

$\square$ mucocartilage $\square$ cell-rich hyaline cartilage

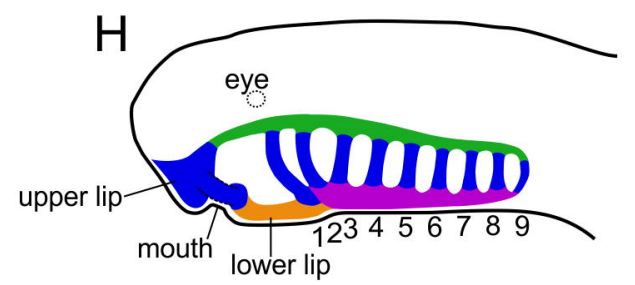

$d l x C,-D,-B$

- $d I x A,-C,-D,-B$

dII $x C,-D,-B$, hand

alxB, hand

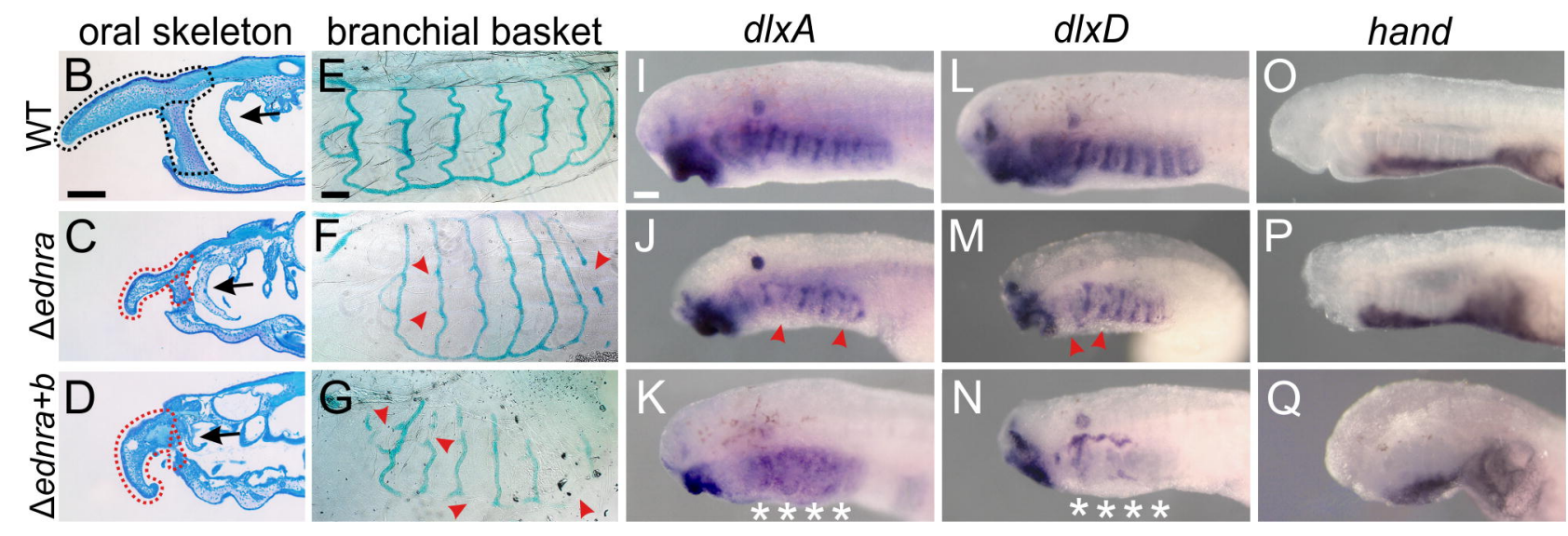


Neurofilament IHC
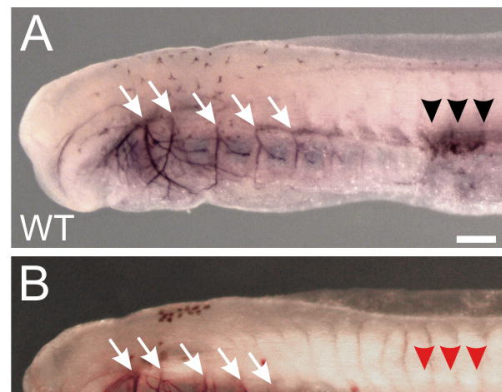

\section{$\Delta$ ednrb}

C

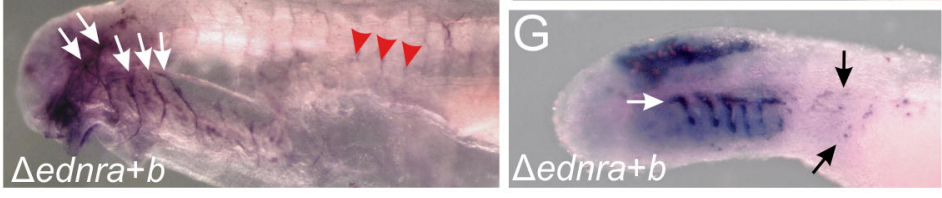

phox2
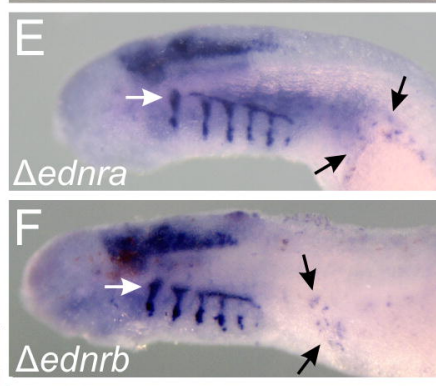
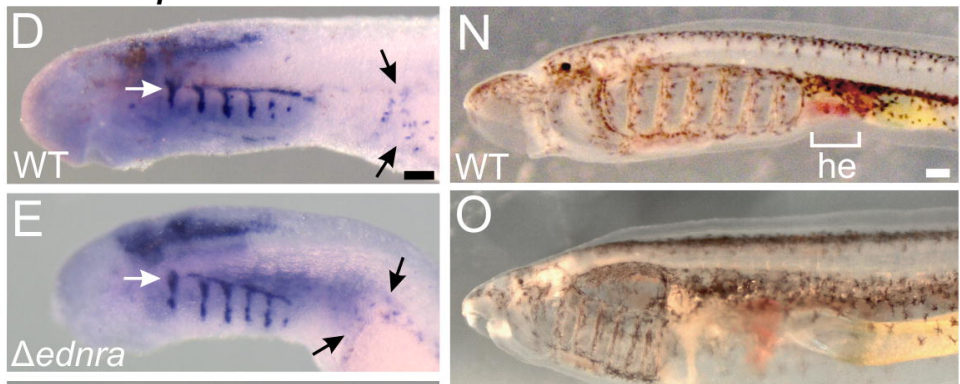

\section{SOXE2}

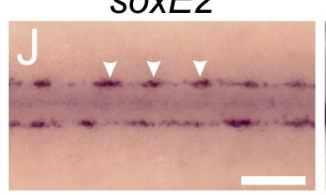

soxB1b

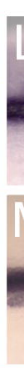

$\triangle e d n A$
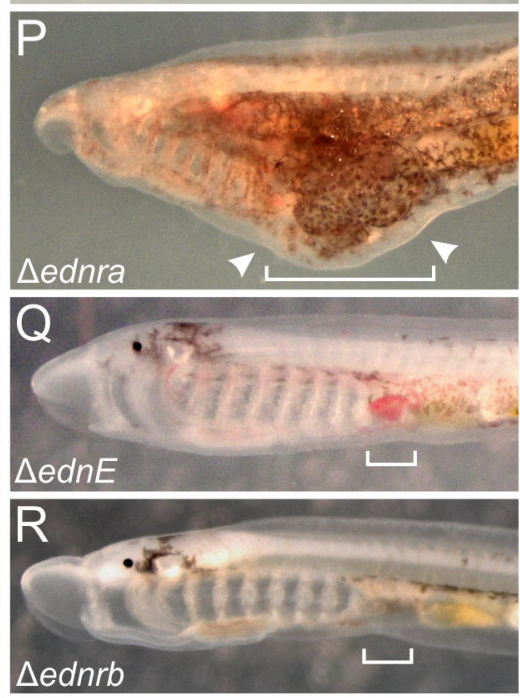

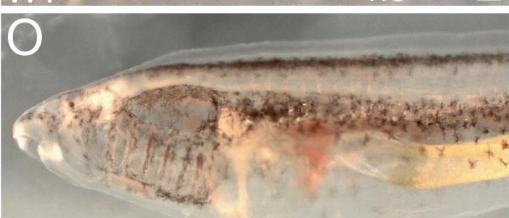


Lancelets

$A^{a}$ Eaf $1-1-10$

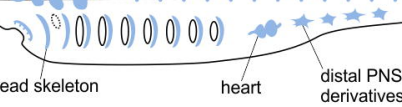
co-opted to neural crest cells

B Late stem vertebrate

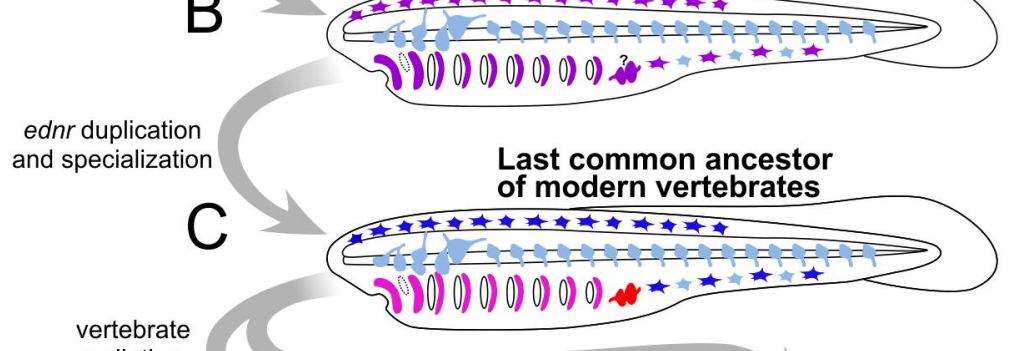

vertebrate adiation

D Cyclostomes (e.g. lamprey)

D' Gnathostomes (e.g. zebrafish) (20.
Endothelin-independent

Endothelin-dependent pre-ednr duplication

Ednra and Ednrb cooperate post-ednr duplication

Ednra-dependent post-ednr duplication

Ednrb-dependen post-ednr duplication
30 living species
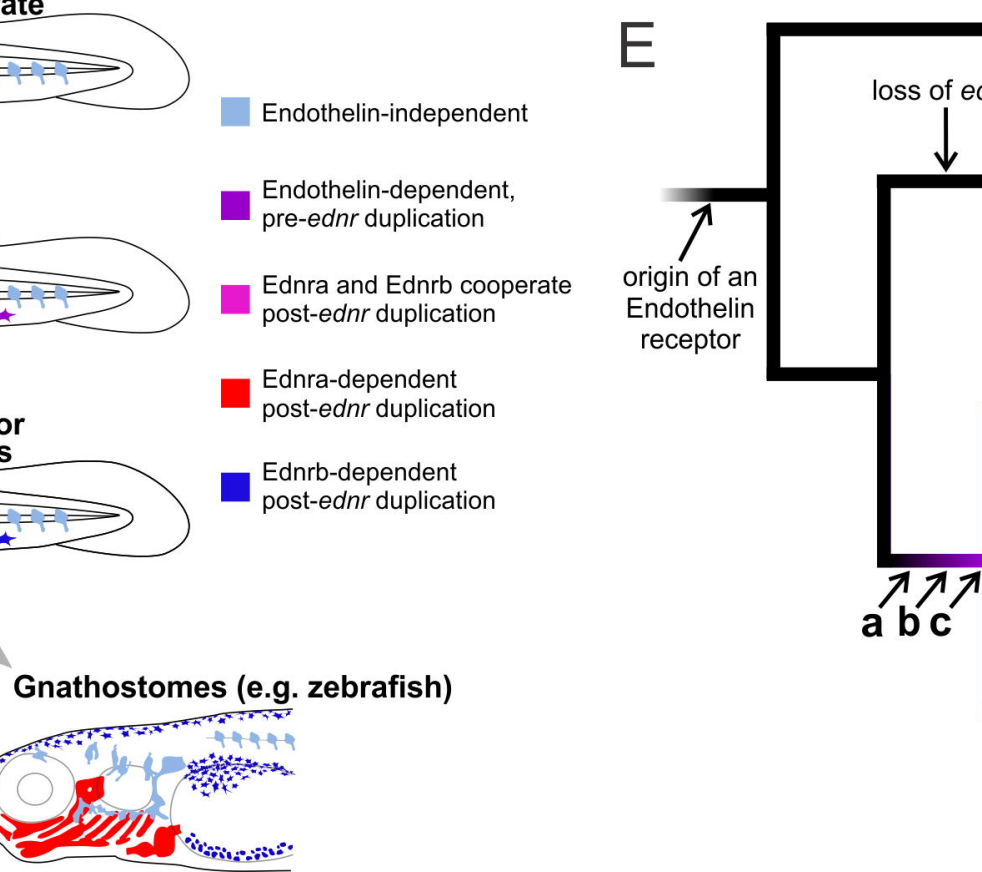

Bony Fishes
Tunicates

$\sim 1,600$ living species

\section{Hagfishes}

78 living species

Lampreys

46 living species

+ multipotent NCCs

Cartilaginous Fishes

1,200 living species

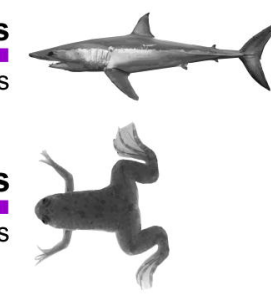

